\title{
LO QUE NOS DICE LA HISTORIA DE LA ENSEÑANZA DE LA BIOLOGÍA EN COLOMBIA -UNA APROXIMACIÓN-
}

Guillermo Chona Duarte, Norma Constanza Castaño Cuellar, Francia María Cabrera Castro, Judith Arteta de Molina, Steyner Valencia Vargas ${ }^{\star}$ y Pablo Antonio Bonilla ${ }^{* *}$

\begin{abstract}
We have few information about the history of teaching Biology in Colombia. From the historic and social analysis, it is possible to recognize the influence of the elements of power and knowledge that come from normative institutions about practice in the teaching of Biology in Colombia. The article describes four historical moments and what we have called mixed knowledge, circumscribed knowledge, biological science and professionalization.
\end{abstract}

\section{PALABRAS CLAVES}

Biología, enseñanza, historia, institucionalización, normatización, profesionalización, saberes circunscritos, saberes mezclados.

\section{INTRODUCCIÓN}

La enseñanza de la Biología en Colombia ha sido influenciada, entre otros factores, por elementos de poder y de saber provenientes de diferentes instituciones normatizadoras. Mediante un análisis histórico de estos elementos, se puede efectuar un acercamiento interpretativo a Lo que han sido y son las prácticas de la enseñanza de la Biología, sus realidades y logros Esta es la pretensión del presente ensayo.

Destacaremos inicialmente, que los estudios sistemáticos sobre la historia de la enseñanza de la Biología en Colombia son escasos. A pesar de ello, es notorio que existen dificultades en el desempeño de los docentes y en la formación científica de los

\footnotetext{
*Profesores de Departamento de Biología. Universidad Pedagógica Nacional.

** Licenciado en Biología GRUPO DE INVESTIGACIÓN ENSENANZA DE LA BIOLOGÍA EN COLOMBIA. 
estudiantes. Al cuestionarnos acerca de esta problemática, suponemos que las enfoques con los que se ha abordado la enseñanza, provienen más de la estructuración de elementos políticos, administrativos y normativos, que del establecimiento de criterios sobre lo que debe ser la función de la enseñanza de la Biología.

La carencia de reflexión y reconocimiento acerca de este sistema de enseñanza nos Lleva a buscar los fundamentos históricos y pedagógicos que le sirven de apoyo. La reflexión que mostramos en este artículo, también tiene este propósito.

Pensamos que debe existir una relación entre la enseñanza y el saber biológico. Nos apoyamos en las consideraciones foucaltianas acerca de la producción de los discursos, donde se señalan que estos se encuentran controlados, seleccionados y redistribuidos por procedimientos que se apoyan en un soporte institucional y se refuerzan por una serie de prácticas, entre las que se encuentra la pedagógica (Foucault, 1983).

Pues bien, en este contexto consideramos la enseñanza como un sistema histórico complejo en el que confluyen elementos producidos por diferentes instituciones normalizadoras y cuya función es asignar, distribuir y adecuar los discursas, pera no sólo esa, sino también, posibilitar el pensamiento dentro de los lineamientos de un saber científico. En nuestra casa, el de la Biología, está circunscrita por objetas, enunciadas, métodos, técnicas, reglas e instrumentas que le son propias y le dan definición.

De otro lado, podemos afirmar que esos saberes y prácticas son eventos que influyen, de una u otra manera, en nuestra cultura y que el encontrar las formas de determinación de esos procesos, nos puede conducir a la búsqueda del fundamento histórico social de las ciencias, de las profesiones, de saberes y prácticas que nos posibilitan nuevos enfoques para entender realidades pasadas y presentes y así, desde esta base, poder establecer Lineamientos para pensar y proyectar lo que puede ser la enseñanza de la Biología.

En este contexto, vislumbramos que la enseñanza de la Biología en Colombia se encuentra mezclada bajo diferentes enfoques en los ámbitos sociales, políticos y económicos. La concepción de enseñanza es aún confusa y en el orden educativo hemos podido detectarla dispersa o refundida ${ }^{1}$ en conceptos como pedagogía y didáctica

Investigaciones en el área de la educación realizadas en los últimos años en Colombia (Díaz y Muñoz. 1990), muestran que la enseñanza no puede ser un catálogo de recomendaciones sobre cómo y qué debe enseñarse, sino a lo sumo una categoría un tanto difusa y no muy bien clarificada, que constituye más bien un campo de problemas donde propósitos relacionados con la pedagogía, la didáctica y aquellas prácticas con las que suele asociarse, son sólo puntos de contraste o niveles de referencia desde los cuales es pasible dar un sentida a tal noción.

Si bien no siempre ha sido evidente la existencia de la enseñanza como problema, hablando en términos históricos (Díaz y Muñoz, 1990), aún se le sigue considerando como un proceso a partir del cual se transmiten saberes de una generación a otra, o como una vía de acceso a la adquisición de nuevos conocimientos.

\footnotetext{
${ }^{1}$ Refundida en el sentido foucaultiano del término.
} 


\section{HISTORICIDAD DE LA ENSENANZA DE LA BIOLOGÍA EN COLOMBIA}

Siguiendo el enfoque histórica social, podemos identificar y aproximarnos a cuatro momentos que han marcada la enseñanza de la Biología en Colombia y a los que hemos denominados Saberes mezclados, saberes circunscritos, ciencia biológica y profesionalización.

\section{SABERES MEZCLADOS}

Los conocimientos Llegados al mal denominado Nuevo Mundo se centran en la instrucción de la historia natural y de la filosofía. Las comunidades religiosas son entonces los centros de difusión y en consecuencia, los preceptores son españoles en su mayoría.

Las ideas, pensamientos y hechos fueron cohesionados, correlacionados y refundidos en el contexto latinoamericono, pues al tener una sola óptica, la de las colonizadores, desde allí se interpretan, narran y establecen los elementos de conocimiento y crítica de las realidades (Rodríguez, 1985).

En Colombia, es notable el caso de la Expedición Botánica que bajo la dirección de José Celestino Mutis agrupó trabajos relacionados con el estudio de flora y fauna, mineralogía, la enseñanza de las matemáticas, física, astronomía, medicina y geografía. Prácticas que se ubicon en el contexto de la Historia natural del Siglo XVIII y a las que no se puede dar el nombre de Biología, puesto que sólo se ocupan de la clasificación y de la descripción y no del análisis de los fenómenos que atañen a lo vivo.

Los problemas descriptivos, los referentes metodológicos y los procedimientos de intervención ${ }^{2}$, utilizados por la Expedición Botánica pueden ser analizados en el contexto de la Historia natural. No podemos hablar allí de Biología como disciplina que se especifica a partir del reconocimiento del fenómeno vida, en la Historia Natural del Siglo XVIII no existe la vida como problema, tampoco la Biología como ciencia.

Otro momento histórico, tiene que ver con la Comisión Corográfica organizada por Agustín Codazzi en 1850, con el objeto de describir el territorio nacional, a manera de continuación de la Expedición Botánica y el establecimiento de las fronteras territoriales, además de las condiciones físicas y políticas, se constituyó así en una actividad cartográfica enmarcada en el contexto de la Historia Natural, pero con una nueva enunciación, la de las Ciencias Naturales.

Se evidencia, en esta forma, que campos del saber que se fueron estableciendo y que permanecieron con ciertas denominaciones en las ambientes culturales, científicos y educativos, no poseen correlación alguna con los términos del desarrolla conceptual, parlas cuales han pasado o se han establecido.

\footnotetext{
2 Procedimientos de intervención en los enunciados, técnicas de reescritura, métodos de trascripción, medios de aproximación, formas de delimitación, transferencias de un campo de aplicación a otro, métodos de sistematización. Cfr. FOUCAULT, M La Arqueología del saber, México. Siglo XXI, 1979, pp 95-96. 


\section{SABERES CIRCUNSCRITOS}

Los conocimientos provenientes de las Ciencias Naturales, fueran incorporados a la vida universitaria a través de la formación de estudiantes en las carreras de Medicina, Jurisprudencia y Filosofía.

Las Ciencias Naturales también presentan un momento específico en la historia colombiana con la creación de las Escuelas de Ciencias Naturales de la Universidad Nacional. Hacia 1868 se crearan distintas cátedras en la Escuela y es importante destacar que ninguna se denominó Biología; se trataba de cursos de Botánica, Zoología, Anatomía Comparada, entre otros (Restrepo,1984).

\section{LA CIENCIA BIOLÓGICA}

A finales del Siglo XIX surgen en el ámbito universitario polémicas en torna al concepto vida como lo constata la obra del Padre Luis Ortiz (1893) y el Estudio sobre el Sistema Evolucionista de Emilio Cuervo (1891).

Paralelas al desarrollo de estas polémicas, encontramos cátedras de Biología en la Universidad Republicona, en el Externado de Colombia y en la Facultad de Filosofía de la Universidad del Rosario. Las cátedras son transitorias y en ellas se discute en torna a las teorías sobre el origen de la vida que venían formulándose y a la teoría celular, reforzada con la experimentación sobre la naturaleza orgánica de los seres vivos, como lo evidencia la obra del Padre Ortíz, ya mencionada.

El establecimiento del Concordato entre la Iglesia Católica y el Estado Colombiano, hace que se controle la difusión de abras científicas, lo cual no permite el desarrolla de las cátedras de Biología, máxime que su temática era ofrecida a juristas, médicos y letrados, como trabaja de discusión ideológico y no de análisis experimental (Restrepo, 1984).

Como es notorio, la Biología aún no encuentra un ámbito institucional firme para su consolidación como práctica científica, pero no par esta desaparece de la historia colombiana. Toma un nuevo papel en la polémica sobre la degeneración de la raza hacia 1920. La Biología participa en su saber con el objeto de evaluar la condición de la población y su posible estado de degeneración y decadencia (Holguín, 1984).

Por otra lado, la Biología participa también en la estrategia de higienizar a la población colombiana, pues desde 1886 la cuestión de la higiene era materia de administración pública. "La necesidad de mejorar las condiciones económicas del país estaban obviamente relacionadas con la higienización y las propuestas de emigración (ambas relacionadas con la degeneración de la raza). Hay que entender que la cuestión de la higiene fue sobre todo un imperativo educativo: educar es higienizar - No es la riqueza ni la prosperidad la garante social de la salud y del gobierno de la población sino más bien la inversa: un conjunto de prácticas que se encabalgan sobre el cuerpo sano asegurando su 
defensa contra la enfermedad, produciría los individuos que serían el fundamento de un arden social más sano y próspero"3.

Mientras en el Sigla XIX en el pensamiento occidental, la Biología define su objeto de conocimiento, rompiendo la continuidad existente entre el estudio de lo animado y lo inanimado, cuando las teorías sobre los seres vivos definen una metodología y unos principios, en la historia colombiana la existencia de la Biología coma discurso se da en las primeras décadas del siglo $\mathrm{XX}$, pero ligada a las condiciones políticas y sociales, no como un discurso institucionalizado objeto de enseñanza. Se trata de una Biología muy ligada a la formación médica y a la acción política del estado.

Hacia 1950, Soriano Lleras (1950): detenta la cátedra de Biología en el Instituto de Filosofía y Letras de la Universidad Nacional. Encontramos que se trata de un discurso mucho más firme sobre la Biología que reconocemos hoy. El curso trata esencialmente los siguientes aspectos:

- Generalidades sobre los seres vivos, su origen y su diferencia con los seres inanimados.

- Teoría de la herencia y especialmente la herencia humana.

- Influencia del medio: nuevamente el problema de la raza, la enfermedad y el sexo.

- El transformismo, sus pruebas y la evolución de la especie humana

- El envejecimiento.

Paralelamente se hacen publicaciones periódicas en el Instituto de Ciencias Naturales de la Universidad Nacional. La Biología se consolida poco a poco en las instituciones educativas con programas, metodologías y proyectos que superan levemente la inmediatez del control político y el desarrollo económico de la nación.

Esto nos lleva a reiterar que solamente es reconocido un discurso en la medida en que éste ha adoptado socialmente unas normas jurídicas y unas prácticas pedagógicas o experimentales.

\section{LA PROFESIONALIZACION}

Una vez que el discurso biológico irrumpe en el ámbito universitario y es reconocido institucionalmente y con la influencia de misiones pedagógicas extranjeras, especialmente la proveniente de Alemania, se inicia la transformación de las escuelas normales en universidades formadoras de profesionales para la enseñanza, cumpliéndose así el proceso de institucionalización de la enseñanza de la biología en el sistema escolar colombiano

Hoy, a partir de la reorganización de las áreas obligatorias y fundamentales del conocimiento en la educación básica, se desdibuja y se descentra la atención a los

\footnotetext{
${ }^{3}$ OSPINA A La higiene y la escuela. Principio de siglo XX en Colombia Tesis de grado Universidad del Valle -Facultad de Filosofía Cali. 1984 
procesos de comprensión e interpretación de lo vivo. El conocimiento biológico se refunde en un enfoque ambientalista, a pesar de que en el contexto universal ha tomado la forma de un poder que permite al hambre transformar la naturaleza y transformarse a si mismo, suscitando discusiones por sus implicaciones éticas, políticas y hasta económicas

\section{A MANERA DE CONCLUSIÓN}

En este breve panorama se resalta, que a pesar de la manifiesta institucionalización de la enseñanza de la biología, aún hoy existen insuficiencias teóricas y falta una práctica pedagógica creada a partir de nuestros propios elementos culturales. Suponemos que se han privilegiado los esquemas de racionalidad de sociedades, donde el saber biológico ha tenido una historia fecunda y la enseñanza ha sido objeto de reflexión permanente Hemos asumido las prácticas de la peor manera, sin crítica, sin reflexión; es urgente construir un espacio para pensar nuestra historia en relación con un sistema de enseñanza que debe crear nuevos modos de pensar la formación de sujetos de pensamiento en un contexto cultural propio.

\section{BIBLIOGRAFIA}

CUERVO, E. 1891. Estudio sobre el sistema evolucionista. Imprenta La Luz. Bogotá.

DIAZ, M. y MUÑOZ, J. A. 1990. Pedagogía discurso y poder. Corprodic. Bogotá.

FOUCAULT. M. 1979. La arqueología del saber. Siglo XXI. México. . 1983. El orden del discurso. Tusquets. Barcelona.

HOLGUíN, H. 1984. Análisis descriptivo de la polémica de la raza en Colombia en 1920. Tesis de grado. Universidad del Valle. Facultad de Filosofía. Cali.

LLERAS, S. 1950. Elementos de Biología. Universidad Nacional de Colombia. Bogotá.

ORTIZ, L. 1893. La vida. Disertación científica del R. P. profesor de Literatura en el Colegio Nacional de San Bartolomé. Imprenta La Luz. Bogotá.

OSPINA, A. 1984. La Higiene y la escuela. Principio de siglo XX en Colombia. Tesis de grado. Universidad del Valle. Facultad de Filosofía. Cali.

RESTREPO, Olga. 1984. Apuntes para una historia social de la Biología en Colombia. Colciencias. Bogotá.

RODRÍGUEZ F., Juan. 1985. El Carnero. Circulo de Lectores. Bogotá. 